\title{
Core of Evidence based Medicine
}

There are known knowns. There are things we know that we know. There are known unknowns. That is to say, there are things that we now know we don't know. But there are also unknown unknowns. There are things we do not know we don't know.'

The above famous statement by Donald Rumsfeld, the $13^{\text {th }}$ and $21^{\text {st }}$ Secretary of Defence for the USA, has been one of the cores to the evidence based medicine movement. In a perfect world, Known Knowns would be facts and evidence based on scientific research and scientific methods. The scientific research carried out so far and being carried out are initially based on investigating the known unknowns. When a researcher carries out a study by developing a hypothesis to be tested, commonly the results obtained will be within a range of known possibilities. At very rare circumstances, the results obtained turn out to be completely different from the expected results. It is known as unknown unknowns. These are the blind spots, variables we have no awareness of and often the most dangerous variables and situations we ever face; because we are very likely to underestimate the number of unknowns that surround us, but they can catch us completely by surprise.

When it comes to the medical field, James Mackenzie explains two kinds of ignorant people; those who don't know and know they don't know and those who don't know and don't know that they don't know. Here the first category people are more likely to be conscious of their defect in knowledge and their first step would be to remedy the defect. On the other hand, the attempts by the second category people in improving matters are less likely to be rational and might be haphazard and ineffective.

Despite the above categories, Evidence based medicine (EBM) emphasizes the concept that one should apply the best evidence from the medical research to the treatment of the patients, preferably the evidence derived from randomized controlled trials. When making a decision about a patient's care, medical professionals often search for, assemble and critically evaluate all the available evidence for the efficacy and safety of therapeutic, rehabilitative and preventive regimens. Mostly the clinicians rely on clinical practice guidelines and recommendations compiled systematically by panels of experts. However, the clinicians must be sure about the validity of the evidence and judgments on which the recommendations are based. Some clinicians might want to reassess the guidelines. This could be done through a simple explicit system which attempts to grade the quality of evidence.

Such evaluation processes mainly focus on assessing the quality of evidence and the strength of a recommendation. The quality of evidence demonstrates the extent to which a clinician can be confident that an estimated effect is correct whereas the strength of recommendation shows the extent to which a clinician could be confident that adherence to 
the particular recommendation will do more good than harm. The recommendations need to be analyzed based on the quality of evidence, trade offs and its applicability whether it is applied to the right population at the right settings. In other words, the EBM could be applied in cycle which is achieved by following the $5 \mathrm{~A}$ technique. Steps are

1. Ask a structured question

2. Acquire relevant evidence

3. Appraise the evidence

4. Applicability of the finding to the patient care (Generalisability and significance to the patient)

5. Act

However, there have been few criticism of evidence based medicine. There is a possibility of bias in the RCTs available as positive controlled trials are more likely to be published rather than the negative results. Further, clinicians need to be very careful when performing literature search and appraisal of all evidence. Institutionally based guidelines cannot always be applied to every clinical situation. Hence, EBM also has limitations despite its advantage of leading to better clinical care.
In summary, EBM addresses the deficiencies in clinical care that rely on expert opinion based on physiological reasoning and unstructured use of evidence and provides in its place a coherent framework for assessing and applying the best available evidence to clinical care decisions. Thus, the best clinicians should evaluate patients' perspectives and biomedicals by developing their excellent communication and clinical skills to make a final diagnosis. They should also be able to tailor the management by giving at least three possible alternatives with reasoning. The final decision on management needs to be the shared one made by both the patients and the clinicians.

\section{Reference:}

1. Davey SG. Commentary: Known knowns and known unknowns in medical research: James Mackenzie meets Donald Rumsfeld, International Journal of Epidemiology. 2016; 45 (6):1747-1748. doi.org/10.1093/ije/dyx02

2. Kamath G. Importance of evidence-based medicine on research and practice. Indian Journal of Anaesthesia. 2016;60(9): 622-625. doi:10.4103/0019-5049.190615.

\section{Ajantha K Editor in Cheif}

